\title{
Right atrial thrombus leading to pulmonary embolism
}

\author{
MARC VAN KUYK, PIERRE MOLS, MARC ENGLERT \\ From the Saint-Pierre University Hospital, Brussels, Belgium
}

SUMMARY Cross sectional and $M$ mode echocardiography detected a large right atrial mass in a 70 year old man five days after admission. Three weeks later he developed acute respiratory failure due to a pulmonary embolism. Necropsy showed that the atrial mass was a thrombus. Thus echocardiography should be performed in patients with pulmonary emboli to examine the right cardiac cavities. The need for emergency surgery could then be assessed.

Although thrombosis of the left side of the heart is fairly common, a right sided thrombosis is a rare echocardiographic finding. The origin of a right sided thrombosis is not often clear, but the clinical course is frequently associated with a pulmonary embolism. We report a patient in whom cross sectional and $M$ mode echocardiography showed a large right atrial mass.

\section{Case report}

A 70 year old man was admitted to hospital in a coma. He had a long history of atrial fibrillation, mitral regurgitation, and chronic heart failure. On physical examination he was comatose and cyanotic, with a left hemiplegia. His blood pressure was $150 / 100 \mathrm{~mm} \mathrm{Hg}$ and his heart rate 92 beats/min and irregular. The jugular venous pressure was raised. On auscultation, there was a grade $3 / 6$ pansystolic murmur indicating mitral regurgitation. Further physical examination showed no abnormalities except for clubbing of the fingers. Biochemical values were normal.

\section{INVESTIGATIONS}

A 12 lead electrocardiogram showed atrial fibrillation, left ventricular hypertrophy with strain, left anterior fascicular block, and ventricular extrasystoles. The chest $x$ ray film showed an enlarged heart without any signs of pulmonary congestion or pulmonary hypertension. Cerebral radionuclide scintigrams and cerebral computed tomograms showed a right sided

Requests for reprints to Dr Marc Van Kuyk, Cardiology Department, Saint-Pierre University Hospital, Rue Haute 322, B-1000 Brussels, Belgium. haemorrhage. A cross sectional echocardiogram performed on the fifth day showed a mitral valve prolapse and a large mobile right atrial mass, probably fixed to the interatrial septum by a peduncle (Fig. 1). During the cardiac cycle this mass moved and appeared to rotate and intermittently obstructed the tricuspid valve without passing into the right ventricle. Overall cardiac dimensions were slightly increased. The cross sectional findings were also present on the $M$ mode echocardiogram (Fig. 2). Mitral regurgitation was also seen using pulsed Doppler. Tricuspid regurgitation was evident by a contrast study after injection of $10 \mathrm{ml}$ intravenous saline. The results of cardiac computed

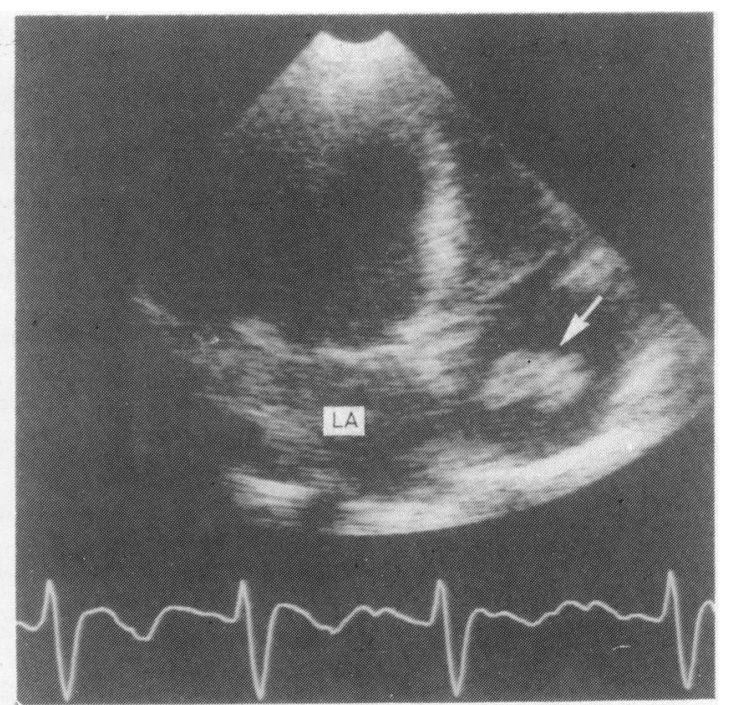

Fig. 1 Cross sectional echocardiogram in the apical four chamber view showing mass in the right atrium (arrow). LA, left atrium. 


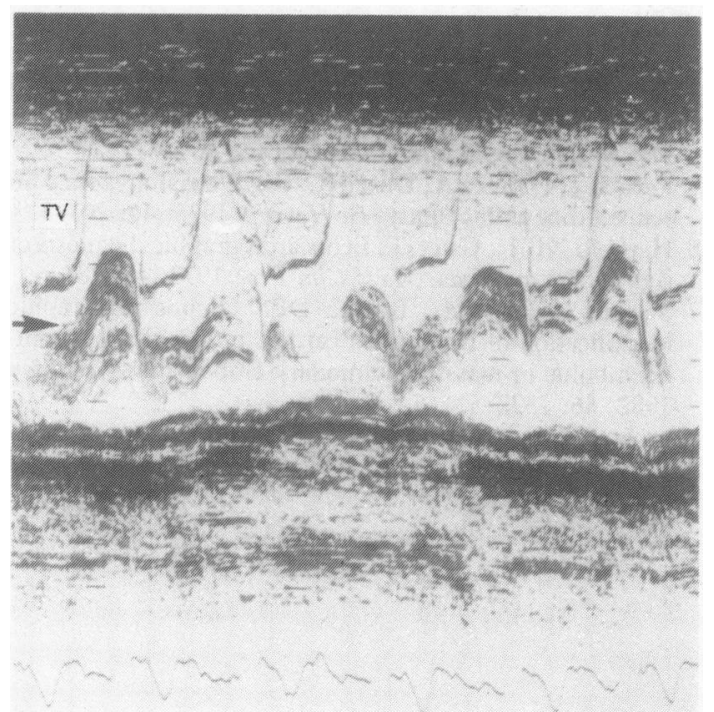

Fig. $2 M$ mode echocardiogram showing the motion of dense echoes (arrow) behind the tricuspid valve (TV).

tomography and indium 111 labelled platelet scintigraphy were normal.

\section{CLINICAL COURSE}

Platelet antiaggregation treatment with acetylsalicyclic acid and dipyridamole was started. His level of consciousness slightly improved but despite intensive physiotherapy the left hemiplegia did not. Cardiologically he remained stable.

Three weeks after admission the patient suddenly developed acute respiratory failure and became intensely cyanosed. Lung scans showed a pulmonary embolism. Cardiac echocardiographic findings remained unchanged. No thrombosis of the lower limbs was evident. The patient became progressively more comatose. Five days after the pulmonary embolism he had a further episode of apnoea and cyanosis, which was fatal. Death was attributed to a recurrent pulmonary embolism.

\section{NECROPSY FINDINGS}

At necropsy no mass was found in the right atrium, but fragments of a small thrombus were present in the right ventricle close to the anterior wall. Thrombi were evident in a left interior and in a right middle branch of the pulmonary artery. No myxomatous tissue was visible on microscopy. The mitral valve was damaged by a sclerotic degeneration. There was no rupture of the papillary muscles. No evidence of deep vein thrombosis was found. The tricuspid valve was normal and there was no atrial tumour. No neoplastic renal disease was evident. The brain showed a right temporopatietal infarction.

\section{Discussion}

Published reports indicate that right atrial masses are rare echographic findings, whose nature is usually determined at necropsy. The clinical diagnosis is usually presumptive. In our patient a prominent eustachian valve could be ruled out by $M$ mode echocardiography as well as by cross sectional echocardiography because the mass was not a linear echo continuous with the inferior vena cava-left atrial junction. ${ }^{1}$ The $M$ mode image could have been consistent with endocarditis of the tricuspid valve ${ }^{2}$ if cross sectional echocardiography had not unequivocally shown an independent mass on the tricuspid valve. Moreover, neither the clinical course nor the laboratory findings favoured this diagnosis. As no evidence of a neoplastic disease could be found, a primary right atrial tumour or the right atrial extension of a Wilms' tumour seemed to be unlikely. ${ }^{3}$

Three further possibilities were a thrombosis of the inferior vena cava extending into the right atrium, ${ }^{4}$ a right atrial myxoma, ${ }^{56}$ or a right atrial thrombus. ${ }^{7}$ The diagnosis of a right atrial myxoma seemed possible since in our patient the mass appeared to be fixed to the interatrial septum. Furthermore, $25 \%$ of all atrial myxomas are found in the right heart. Neither the clinical nor the biochemical findings, which were normal, were consistent with the diagnosis. ${ }^{5}{ }^{6}$ Finally, echocardiography cannot distinguish between an isolated right atrial thrombus, the atrial extension of an inferior vena caval thrombosis, or the embolisation of a peripheral vein thrombosis, even though in the last two instances a coiled mass is usually evident. Since in our patient necropsy did not show any inferior vena caval thrombosis the thrombus may have been formed in another vein.

Whatever the nature of a right atrial mass it usually generates either a microembolism or a massive pulmonary embolism and causes a deterioration in respiratory function which may eventually lead to death. Thus in any patient with a pulmonary embolism the right cardiac cavities should be examined by echocardiography. When a right heart thrombus is the cause of pulmonary microembolisation, the bulk of the mass is probably left in the atrium. The cross sectional echocardiographic findings could therefore help in deciding whether or not to operate immediately.

We thank Mrs Josyane Bourbigot for her technical assistance, and Dr Nicole Naeije for her help in preparing the manuscript. 


\section{References}

1 Bommer WJ, Kwan OL, Mason DT, De Maria AN. Identification of prominent eustachian valves by $M$-mode and two-dimensional echocardiography: differentiation from right atrial masses [Abstract]. Am $\mathcal{f}$ Cardiol 1980; 45: 402.

2 Come PC, Kurland GS, Vine HS. Two dimensional echocardiography in differentiating right atrial and tricuspid valve mass lesions. Am $\mathcal{F}$ Cardiol 1979; 44: 1207-12.

3 Farooki ZQ, Henry JG, Green EW. Echocardiographic diagnosis of right atrial extension of Wilms' tumor. Am. 7 Cardiol 1975; 36: 363-7.

4 Broadbent JC, Tajik AJ, Wallace RB. Thrombus of inferior vena cava presenting as right atrial tumour. $\mathcal{f}$ Thorac Cardiovasc Surg 1976; 72: 422-6.

5 Pernod J, Piwnica A, Duret JC. Right atrial myxoma: an echocardiographic study. Br Heart f 1978; 40: 201-3.

6 Harbold NB Jr, Gau GT. Echocardiographic diagnosis of right atrial myxoma. Mayo Clin Proc 1973; 48: 284-6.

7 Starkey IR, de Bono DP. Echocardiographic identification of right sided cardiac intracavitary thromboembolus in massive pulmonary embolism. Circulation 1982; 66: 1322-5. 